\title{
Using The Cancer Genome Atlas (TCGA) as an inquiry tool in the undergraduate classroom
}

William Hankey ${ }^{1}$, Nicholas Zanghi ${ }^{2}$, Kenzy $\mathrm{Crow}^{2}$, Whitney Dow ${ }^{2}$, Austin Kratz ${ }^{2}$, Ashley Robinson ${ }^{2}$, Meaghan Robinson ${ }^{2}$, Verónica A. Segarra ${ }^{2 *}$

${ }^{1}$ Duke University, United States, ${ }^{2}$ High Point University, United States

Submitted to J ournal:

Frontiers in Genetics

Specialty Section:

Evolutionary and Genomic Microbiology

Article type:

Perspective Article

Manuscript ID:

573992

Received on:

18 J un 2020

Frontiers website link:

www. frontiersin.org 


\title{
Conflict of interest statement
}

The authors declare that the research was conducted in the absence of any commercial or financial relationships that could be construed as a potential conflict of interest

\section{Author contribution statement}

WH and VAS designed the activities outlined in this manuscript. WH, NZ, KC, WD, AK, AR, MR, and VAS implemented the activities, wrote, and revised the manuscript. All authors accept and are in agreement with the content of the manuscript.

\section{Keywords}

bioinformatics, Cancer, Genomics, Cancer genomics, Undergraduate teaching and learning

\begin{abstract}
Word count: 132

Undergraduate students in the biomedical sciences are often interested in future health-focused careers. This presents opportunities for instructors in genetics, molecular biology and cancer biology to capture their attention using lab experiences built around clinically relevant data. As biomedical science in general becomes increasingly dependent on high-throughput data, well-established scientific databases such as TCGA have become publicly available tools for medically relevant inquiry. The best feature of this database is that it bridges the molecular features of cancer to human clinical outcomes, allowing students to see a direct connection between the molecular sciences and their future professions. We have developed and tested a learning module that leverages the power of TCGA datasets to engage students to use the data to generate and test hypotheses and to apply statistical tests to evaluate significance.
\end{abstract}

\section{Contribution to the field}

While undergraduate access to clinical research experiences is limited, the biomedical sciences is becoming increasingly dependent on high-throughput data, well-established scientific databases such as TCGA that have become publicly available tools for medically relevant inquiry (Cancer Genome Atlas Network, 2012; Cerami et.al., 2012; Gao et. al., 2013). Moreover, these databases are increasingly being recognized as resources available for undergraduate teaching (Coughlan, 2020). This Perspective article describes an undergraduate bioinformatics exercise we designed to help fill this gap.

\section{Funding statement}

No funding was received to carry out this research.

\section{Ethics statements}

\section{Studies involving animal subjects}

Generated Statement: No animal studies are presented in this manuscript.

\section{Studies involving human subjects}

Generated Statement: No human studies are presented in this manuscript.

\section{Inclusion of identifiable human data}

Generated Statement: No potentially identifiable human images or data is presented in this study.

\section{Data availability statement}

Generated Statement: All datasets presented in this study are included in the article/ supplementary material. 
1 Using The Cancer Genome Atlas (TCGA) as an inquiry tool in the

2 undergraduate classroom

3 William Hankey ${ }^{*}$, Nicholas Zanghi ${ }^{2}$, Kenzy Crow ${ }^{2}$, Whitney Dow ${ }^{2}$, Austin Kratz ${ }^{3,4}$, Ashley

4 Robinson $^{2}$, Meaghan Robinson ${ }^{2}$, and Verónica A. Segarra ${ }^{2 *}$

$5{ }^{1}$ Department of Pathology, Duke Cancer Center, Duke University, Durham, NC, USA

$6 \quad{ }^{2}$ Department of Biology, High Point University, High Point, NC, 27268 USA

$7 \quad{ }^{3}$ Department of Chemistry, High Point University, High Point, NC, 27268 USA

$8 \quad{ }^{4}$ Department of Physics, High Point University, High Point, NC, 27268 USA

* Correspondence:

11 Co-corresponding Authors

12 william.hankey@duke.edu

13 vsegarra@highpoint.edu

14 Keywords: The Cancer Genome Atlas, 1 , Science Pedagogy 2 , Bioinformatics, Cancer $_{4}$, 15 Genomics $_{5}$, Cancer Genomics.

Abstract

Undergraduate students in the biomedical sciences are often interested in future health-focused careers. This presents opportunities for instructors in genetics, molecular biology and cancer biology to capture their attention using lab experiences built around clinically relevant data. As biomedical science in general becomes increasingly dependent on high-throughput data, well-established scientific databases such as TCGA have become publicly available tools for medically relevant inquiry. The best feature of this database is that it bridges the molecular features of cancer to human clinical outcomes - allowing students to see a direct connection between the molecular sciences and their future professions. We have developed and tested a learning module that leverages the power of TCGA datasets to engage students to use the data to generate and test hypotheses and to apply statistical tests to evaluate significance.

\section{Introduction}

While many undergraduates are interested in becoming medical doctors and declare "pre-med" early in their academic careers, it is predicted that by 2032 the United States will face a shortage of between 46,900 and 121,900 physicians (Dall et. al., 2019). One of the likely factors contributing to this projected shortage is the high attrition rates of undergraduates from the premedical academic track, especially those from underrepresented backgrounds in STEM (Lin et. al., 2013). In fact, many of the empirical studies recorded in the scientific literature related to undergraduate premedical students are focused on documenting and better understanding attrition from the premedical track which has been shown to disproportionally affect underrepresented groups in STEM (Lin et. al., 2013). High attrition rates in undergraduate premedical tracks has been found to be influenced by a variety of factors including loss of interest and negative experiences in required courses (Lin et. al., 2013). 
39 Student interest and persistence in STEM careers can be increased and strengthened through

40 participation in Course-based Undergraduate Research Experiences (CUREs) as part of the

41 curriculum, especially in populations from underrepresented backgrounds (Estrada et. al., 2017).

42 These findings suggest that one of the ways in which student persistence in undergraduate premedical programs can be increased is through relevant CURE experiences that highlight clinically relevant data and its applications.

While undergraduate access to clinical research experiences is limited, the biomedical sciences is becoming increasingly dependent on high-throughput data, well-established scientific databases such as TCGA that have become publicly available tools for medically relevant inquiry (Cancer Genome Atlas Network, 2012; Cerami et.al., 2012; Gao et. al., 2013). Moreover, these databases are increasingly being recognized as resources available for undergraduate teaching (Coughlan, 2020).

Furthermore, there is currently a need for physicians and health professionals to recognize and use the power of cutting edge genomics to inform diagnosis and treatments for their patients (Rubanovich et. al., 2018). Through the use of clinically relevant genomic datasets like the ones found in TCGA in the undergraduate classroom, we can raise awareness for the relevance of these resources in medicine early on in the training of these individuals (Schoenborn et.al, 2019).

We have developed and tested a learning module that leverages the power of TCGA datasets to engage students in clinical research in the context of cancer-a human disease that is of universal relevance. Our module allows students to, not only generate and test hypotheses with clinical relevance, but also apply statistical tests to evaluate significance. To the extent that we can deepen the degree to which experiences like these are developed, we can be in a better position to foster student interest and persistence at the level of undergraduate premedical tracks.

Methodology

Accessing TCGA Datasets.

TCGA data were accessed by the course instructor through cBioportal (https://www.cbioportal.org/), a widely-used web interface that provides access to public cancer genomics datasets (Cancer Genome Atlas Network, 2012; Cerami et.al., 2012; Gao et. al., 2013). To acknowledge that TCGA datasets are updated over time with additional samples and details, we note that these data were accessed on January $2^{\text {nd }}, 2020$ and were current as of that date. Breast cancer was selected as a focus because of the increased likelihood for the intended audience members to make personal connections to a highly prevalent cancer type with a significant impact on human health, and because of the convenience of introducing the genomic data starting with familiar genes such as $B R C A 1, B R C A 2$ and the gene encoding p53 (TP53) that had previously been discussed in the lecture component of the class. TCGA was chosen as a data source for the combination of high-quality genomic and associated clinical data characteristic of TCGA datasets in general and the high sample size of the available datasets. The TCGA Breast Invasive Carcinoma dataset associated with the 2015 publication in Cell (Ciriello et.al., 2015) was specifically chosen from among the four available TCGA Breast Invasive Carcinoma datasets because of its combination of mutation data and, copy number alteration data, as well as its inclusion of stage among the clinical data variables. The total number of tumor samples in this dataset was 817 .

While mutation and copy number data were available in the dataset for more than 20,000 genes, a more focused subset of sixteen total genes was selected to provide to the students. This subset was narrow enough facilitate visualization of the complete dataset and analysis by first-time 
bioinformaticists in Microsoft Excel, but diverse enough to include examples fitting several different patterns. The list began with well-known cancer-associated genes previously discussed in the course (BRCA1, BRCA2, TP53, then added genes that were among the most frequently targeted in breast cancer by known pathogenic mutations (PIK3CA, CDH1, GATA3, MAP3K1, KMT2C, AKT1), amplifications (MYC, CCND1, ERBB2), or deletions $(R B 1, P T E N)$. These high-frequency targets of mutations and copy number alterations were identified by selecting the dataset of interest (Cancer Genome Atlas Network, 2012) from the cBioportal menu and using the Explore Selected Studies function to view the Summary of findings. The genes encoding $\beta$-actin $(A C T B)$ and hemoglobin subunit $\beta(H B B)$ were added to the list in order to function as recognizable negative control genes generally not associated with cancer. Once the list of sixteen breast cancer-relevant genes and controls was determined, the sixteen gene names were entered as a list into the cBioportal website to access genomics data for this subset using the Query by Gene function. For each gene of interest, genetic mutation data and copy number alteration data were separately accessed for all 817 tumors in the dataset from the Download section of the site, selecting the Tab Delimited Format option. Clinical data were accessed through the cBioportal site using the Explore Selected Studies function and the Clinical Data tab. A limited subset of clinical characteristics were downloaded, with each characteristic chosen to help illustrate a different point or to enable the students to test a different hypothesis. The majority of clinical variables were categorical, facilitating the use of $2 \times 2$ tables to test association between the clinical category and the status of a gene as mutated / unmutated, etc. The 15 characteristics were Informed Consent by Patient (Yes/No), Diagnosis Age, Cancer Type, Race Category, Ethnicity Category, Sex, Disease Stage (I-IV), Treatment Outcome (Living DiseaseFree / Living with Tumor / Recurred or Progressed / Deceased), Time from Treatment to Recurrence (Months), Time from Treatment to Death (Months), Time from Treatment to Most Recent Contact (Months), ER Status (by Staining), PR Status (by Staining), HER2 Status (by Staining), and Total Number of Mutations. Similar to the mutation and copy number data files, the clinical data were arrayed so that the clinical variables were each assigned a different column, while the 817 tumors were each assigned a different row (Appendix 1).

\section{Combining Genetic and Clinical TCGA Data in Microsoft Excel (Appendix 1).}

Initially, the separate Mutations and Copy-Number Alterations files were integrated into a single Excel file by alphabetizing the list of samples in each file by Patient ID and integrating the columns along matching rows. The instructor then sought to integrate the mutation status and the copy number status into a single column for each gene, stating only the change in that gene most relevant to the disease. For example, if the Copy Number Alteration column for TP53 listed the gene as Amplified in a particular tumor, while the Mutations column for TP53 listed it as a known Pathogenic Mutant in that same tumor, the merging of those two columns into one TP53 Status column listed it as Pathogenic Mutant for that tumor. On the other hand, if the Copy Number Alteration column for TP53 listed the gene as Amplified in a particular tumor, while the Mutations column for TP53 listed it as a Mutant of Unknown Significance in that same tumor, the merging of those two columns into one TP53 Status column listed it as Amplified for that tumor. The resulting Excel file containing gene status data was then integrated with the Clinical Data file into a single Excel file by alphabetizing the list samples in each file by Patient ID and integrating them along matching rows. The resulting file contained 16 columns of genetic data and 15 columns of clinical data, with 817 rows of tumor samples, each representing a different patient (Appendix 1).

\section{Generation of the Worksheet}


The instructor designed an assignment to introduce students to the kinds of research hypotheses that are testable using the combination of genetic and clinical data. The initial assignment was generated in the form of a worksheet (Appendix 2), which consisted of five different tables. Categorical clinical and/or genetic characteristics were listed along the $\mathrm{x}$ - and $\mathrm{y}$-axes, and students were asked to count how many tumors from the dataset possessed each combination of characteristics. Students first determined how many of the patients classified as Living Disease-Free, Living with Tumor, Recurred or Progressed, and Deceased were diagnosed with Stage I vs. II vs. III vs. IV tumors. This comparison of stage and outcome was selected to illustrate a well-known clinical association and presented students with an opportunity to test whether the counts matched their expectations. Students then determined how many of the patients classified as Living Disease-Free, Living with Tumor, Recurred or Progressed, and Deceased harbored vs. did not harbor pathogenic mutations / deletions in $p 53, B R C A 1$ or $B R C A 2$. Students were already familiar with all three genes as wellknown tumor suppressors in breast cancer, and were able to formulate hypotheses about how mutations in each gene might associate with clinical outcome. In the final table, students were asked to the total number of tumors with pathogenic mutant, mutant of unknown significance, amplified, and deleted genotypes, for each of the sixteen genes. Since most of these genes were less familiar, students would have the opportunity to collect the data without bias, and then to use them to form a hypothesis about each gene's status as an oncogene, tumor suppressor gene, or neither.

\section{Generation of Instructions for Sorting Tumors in Microsoft Excel}

Students came into the assignment with heterogeneous backgrounds using Microsoft Excel for similar tasks, and were provided with general instructions to help them complete the worksheet (Appendix 3). The Sort \& Filter function in Excel was recommended as a critical tool for organizing data into subsets according to a particular genomic or clinical characteristic. Within each subset, students were recommended to count occurrences of the associated characteristic using the COUNTIF function in combination with quotation marks around the text of interest.

\section{Generation of a Microsoft Excel file to Support Statistical Analysis}

As a follow-up assignment, students were asked to use the counts data from their completed worksheet to generate one hypothesis about the association of two variables. They would then construct a $2 \times 2$ table and perform a test for statistically significant association. The chi-square test of independence was recommended as an applicable statistical test that can be performed using Excel. To facilitate their introduction to this statistical test, a template Excel file was constructed into which the students could enter their $2 \times 2$ table (Appendix 4). The file would then use these observed counts to calculate the expected counts, determine the test statistic, and generate a $p$-value.

\section{Classroom Implementation}

The documents/data described above (also see Supplemental Materials/Appendices) were used to create and implement a bioinformatics laboratory experience during two 3-hour lab periods near the conclusion of an upper-level undergraduate Cancer Biology course. This activity can also be implemented in a bioinformatics or genetics course and is particularly well suited to be implemented remotely in the context of online teaching.

167 Students were introduced to the data subset of interest, including the kind of information each 168 column and row contained (Appendix 1). 
169 Step 2: Students complete a worksheet composed of $2 \times 2$ tables that measure associations between

170 presencelabsence of a mutation and categorical clinical phenotypes

171 Students were given the opportunity to increase their familiarity of the dataset of interest (Appendix 172 1) by completing an Excel worksheet (Appendix 2) that required them to identify the data relevant to 173 different categories. To help students sift through the data, they were provided with tips for sorting 174 tumor data in Excel (Appendix 3).

175 Step 3: Students articulate a new association of interest to test (research question), create/complete 176 the appropriate $2 \times 2$ tables, and calculate statistical significance of association

177 Using the data as a guide, students were given the opportunity to come up with their own association 178 or research question to test (Table 1). Students had to examine the data provided and decide which 179 two categorical variables they wanted to use to test an association. Students were introduced to the 180 Chi Square Test of Significance and its relevance to categorical data. To facilitate students 181 performing the relevant statistics, an Excel file template was provided (Appendix 4). Before

182 beginning this portion of the assignment, the instructor demonstrated the process from selection of an 183 association of interest and $2 \times 2$ table construction, all the way to statistical analysis.

\section{Discussion}

185 While, at first, students had difficulty managing the large amount of information that was provided, sharing with them strategies to sort and count data using Excel helped them gain confidence in using the dataset to complete Steps 2 and 3 described above. In fact, all students were ultimately able to get perfect grades on their practice worksheets (Appendix 2).

Table 1 provides representative research questions that were answered using the breast cancer tumor data available. In general, many of the associations tested were not statistically significant, this is likely due to, as noted by others, shortcomings of the dataset (Huo et.al, 2017; Liu et. al., 2018). For example, clinical annotation of TCGA datasets with patient survival and treatment outcomes is incomplete - follow-up times are short (TCGA only stayed in touch with clinicians regarding their patients' clinical outcomes for a short period of time) and data is unclear at times about what the cause of death actually was (may not have been cancer) (Huo et.al, 2017). Moreover, breast cancer is a less aggressive cancer type, and can take 10 years or more to recur (Liu et. al., 2018). So given the relatively short window of follow-up time during which TCGA outcomes were measured (reported by clinicians following up on their patients), overall survival is not a suitable clinical outcome to use (Liu et. al., 2018). Overall survival is also complicated by other causes of death besides breast cancer. Disease-free survival / recurrence might have been a better endpoint to use (Liu et. al., 2018).

All in all, we find this is an effective way for students to experience clinically relevant inquiry in the classroom. This bioinformatics activity can also be expanded by having the students selecting the cancer of interest and pulling relevant data from TCGA.

\section{Conflict of Interest}

205 The authors declare that the research was conducted in the absence of any commercial or financial 206 relationships that could be construed as a potential conflict of interest. 
208 WH and VAS designed the activities outlined in this manuscript. WH, NZ, KC, WD, AK, AR, MR, and VAS implemented the activities, wrote, and revised the manuscript. All authors accept and are in

210 agreement with the content of the manuscript.

\section{Funding}

212 None.

\section{Acknowledgments}

214 We thank the High Point University, its Wanek School of Natural Sciences and its Department of

215 Biology for the resources to carry out this work.

\section{Supplementary Material}

217 Appendix 1: TCGA Breast Cancer Data Subset Sheet: Microsoft Excel file

218 Appendix 2: Instructional Student Data Analysis Worksheet: Microsoft Excel file

219 Appendix 3: Tips for Sorting Tumors in Microsoft Excel

220 Appendix 4: Microsoft Excel file to Support Statistical Analysis

\section{Data Availability Statement}

222 The datasets generated and analyzed for this publication can be found in the supplementary materials provided in the form of Microsoft Excel files.

\section{References}

225 Cancer Genome Atlas Network. Comprehensive molecular portraits of human breast tumours.

226 (2012). Nature. 490, 61-70. doi: 10.1038/nature11412.

227 Cerami E., Gao J., Dogrusoz U., Gross B.E., Sumer S.O., Aksoy B.A., Jacobsen A., Byrne C.J., Heuer M.L., Larsson E., Antipin Y., Reva B., Goldberg A.P., Sander C., Schultz N. The cBio cancer genomics portal: an open platform for exploring multidimensional cancer genomics data. (2012).

231 Ciriello G., Gatza M.L., Beck A.H., Wilkerson M.D., Rhie S.K., Pastore A., Zhang H., McLellan M.,

237 Coughlan, T. (2020). The use of open data as a material for learning. Educ. Technol. Res. Dev. 68, $238 \quad 383-411$.

239 Dall, T., West, T., Chakrabarti, R., Reynolds, R., Iacobucci, W. (2019). The complexities of 240 physician supply and demand: projections from 2015 to 2032. Washington, DC: I. H. S. Markit Ltd. 
242 Estrada, M., Burnett, M., Campbell, A. G., Campbell, P. B., Denetclaw, W. F., Gutiérrez, C. G., ... \&

243 Okpodu, C. M. (2016). Improving underrepresented minority student persistence in STEM. CBE Life

244 Sci. Educ. 15, es5. doi: 10.1187/cbe.16-01-0038

245 Gao J., Aksoy B.A., Dogrusoz U., Dresdner G., Gross B., Sumer S.O., Sun Y., Jacobsen A., Sinha

246 R., Larsson E., Cerami E., Sander C., Schultz N. (2013). Integrative analysis of complex cancer

247 genomics and clinical profiles using the cBioPortal. Sci. Signal. 6, 11. doi:

248 10.1126/scisignal.2004088. PMID: 23550210

249 Huo D., Hu H., Rhie S.K., Gamazon E.R., Cherniack A.D., Liu J., Yoshimatsu T.F., Pitt J.J.,

250 Hoadley K.A., Troester M., Ru Y., Lichtenberg T., Sturtz L.A., Shelley C.S., Benz C.C., Mills G.B.,

251 Laird P.W., Shriver C.D., Perou C.M., Olopade O.I. (2017). Comparison of Breast Cancer Molecular

252 Features and Survival by African and European Ancestry in The Cancer Genome Atlas. JAMA

253 Oncol. 3:1654-1662.

254

255 Lin, K. Y., Parnami, S., Fuhrel-Forbis, A., Anspach, R. R., Crawford, B., and De Vries, R. G. (2013).

256 The undergraduate premedical experience in the United States: a critical review. Int. J. Med. Educ. 4, $257 \quad 26-37$.

258 Liu, J., Lichtenberg, T., Hoadley, K. A., Poisson, L. M., Lazar, A. J., Cherniack, A. D., ... \& Omberg, 259 L. (2018). An integrated TCGA pan-cancer clinical data resource to drive high-quality survival 260 outcome analytics. Cell, 173(2), 400-416.

Rubanovich, C. K., Cheung, C., Mandel, J., \& Bloss, C. S. (2018). Physician preparedness for big genomic data: a review of genomic medicine education initiatives in the United States. Hum. Mol. Genet. 27(R2), R250-R258.

265 Schoenborn, P., Osborne, R., Toms, N., Johnstone, K., Milsom, C., Muneer, R., ... \& Belshaw, R. 266 (2019). OncoSim and OncoWiki: an authentic learning approach to teaching cancer genomics. BMC 267 Med. Educ. 19, 407. 
Table 1. Representative research questions answered by students using TCGA Breast Invasive Carcinoma datasets. For Step 3 in Classroom Implementation, students articulate a new association of interest to test (research question), create/complete $2 \times 2$ tables, and calculate its statistical significance. Shown in this table are representative research questions (associations being tested), including the categorical variables being tested and the determined variable of the association. Shaded rows denote associations that were not independent from each other as evidenced by a pvalue less or equal than 0.05 .

\begin{tabular}{|c|c|c|}
\hline Research question & Categorical variables being compared & p-value \\
\hline $\begin{array}{l}\text { Are pathogenic } P I K 3 C A \text { gene mutations } \\
\text { associated with poor clinical outcomes } \\
\text { (not living disease-free) for breast } \\
\text { cancer? }\end{array}$ & $\begin{array}{l}\text { Wildtype PIK3CA vs. pathogenic } \\
\text { mutations in PIK3CA } \\
\text { Good clinical outcome (living disease- } \\
\text { free) vs. } \\
\text { Poor clinical outcome (not living } \\
\text { disease-free) }\end{array}$ & 0.24 \\
\hline $\begin{array}{l}\text { Is the wildtype } B R C A 2 \text { gene associated } \\
\text { with good (living disease-free) breast } \\
\text { cancer clinical outcomes? }\end{array}$ & $\begin{array}{l}\text { Wildtype } B R C A 2 \text { vs. pathogenic mutant } \\
B R C A 2 \\
\text { Good clinical outcome (living disease- } \\
\text { free) vs. } \\
\text { Poor clinical outcome (not living } \\
\text { disease-free) }\end{array}$ & 0.93 \\
\hline $\begin{array}{l}\text { Are } B R C A 1 \text { gene tumor mutations } \\
\text { associated with poor (not living disease- } \\
\text { free) breast cancer outcomes? }\end{array}$ & $\begin{array}{l}\text { Wildtype } B R C A 1 \text { vs. mutated BRCA1 } \\
\text { gene } \\
\text { Good clinical outcome (living disease- } \\
\text { free) vs. } \\
\text { Poor clinical outcome (not living } \\
\text { disease-free) }\end{array}$ & 0.60 \\
\hline $\begin{array}{l}\text { Are pathogenic TP } 3 \text { mutations } \\
\text { associated with more advanced (Stages } \\
\text { II/III/IV) stages of cancer? }\end{array}$ & $\begin{array}{l}\text { Wildtype } T P 3 \text { vs. pathogenic } T P 3 \text { gene } \\
\text { mutations } \\
\text { Early (Stages I) vs. advanced stages of } \\
\text { cancer (Stages II/III/IV) }\end{array}$ & 0.14 \\
\hline $\begin{array}{l}\text { Are pathogenic } B R C A l \text { mutations } \\
\text { associated with breast cancer recurrence? }\end{array}$ & $\begin{array}{l}\text { Wildtype } B R C A 1 \text { vs. pathogenic } B R C A 1 \\
\text { mutants } \\
\text { Good clinical outcome (living disease- } \\
\text { free) vs. } \\
\text { Poor clinical outcome (living but tumor } \\
\text { recurred/progressed) }\end{array}$ & 0.01 \\
\hline
\end{tabular}


TCGA in the classroom

\begin{tabular}{|l|l|l|}
\hline $\begin{array}{l}\text { Are patients living disease-free more } \\
\text { likely to have been diagnosed early stage } \\
\text { breast cancer (Stages I/II)? }\end{array}$ & $\begin{array}{l}\text { Living disease-free vs. Not living } \\
\text { disease free } \\
\text { Early stage (Stages I/II) vs. late stage } \\
\text { (III/IV) cancer }\end{array}$ & $2 \times 10^{-6}$ \\
\hline
\end{tabular}

